Cinémas

Revue d'études cinématographiques

Journal of Film Studies

\title{
Notes sur les rapports entre l'Office catholique international du cinéma et la filmologie
}

\section{François Albera}

Volume 19, numéro 2-3, printemps 2009

La filmologie, de nouveau

URI : https://id.erudit.org/iderudit/037549ar

DOI : https://doi.org/10.7202/037549ar

Aller au sommaire du numéro

Éditeur(s)

Cinémas

ISSN

1181-6945 (imprimé)

1705-6500 (numérique)

Découvrir la revue

Citer cette note

Albera, F. (2009). Notes sur les rapports entre l'Office catholique international du cinéma et la filmologie. Cinémas, 19(2-3), 103-112.

https://doi.org/10.7202/037549ar d'utilisation que vous pouvez consulter en ligne. 


\section{Notes sur les rapports entre l'Office catholique international du cinéma et la filmologie}

\section{François Albera}

La Revue internationale du cinéma (1949-1956), organe de l'Office catholique international du cinéma (OCIC), possède des objets et des préoccupations qui croisent en plus d'un point la filmologie sur fond de convictions communes: importance de l'éducation, de la jeunesse, du cinéma dans la société et de ses pouvoirs de suggestion, danger de la propagande; etc. Même affirmation selon laquelle «le pouvoir idéomoteur des images, la persistance dans l'imagination des spectacles vus sont d'autant plus forts qu'ils relèvent d'une activité inconsciente de l'esprit» (J.-P. Chartier, n 2, 1949, p. 25) ou encore que "l'influence exercée par le cinéma sur la façon de vivre et de penser de la masse des spectateurs place le septième art à l'avant-garde des moyens de propagande de l'âge moderne" (J. Romero Marchent, $n^{\circ}$ 9, 1951, p. 20).

Sauf que l'OCIC tend, bien entendu, à promouvoir un type de cinéma et une vision du monde et que c'est à ce titre que la maîtrise des mécanismes de la perception filmique est, pour lui, nécessaire.

Quoi qu'il en soit le projet filmologique intéresse d'emblée les rédacteurs de la revue et les responsables de l'OCIC qui se préoccupent également de voir "la pensée "spiritualiste" [ne pas] se désintéresse[r] de recherches qui peuvent avoir des répercussions très profondes sur ce qu'on pourrait appeler l' "hygiène mentale" des peuples entiers. Nous ne pourrions trop encourager les savants catholiques de tous les pays du monde, non seulement de suivre avec intérêt ces travaux, mais aussi d'y participer activement par leur propre investigation" $\left(\mathrm{n}^{\circ} 3,1949\right.$, p. 67).

La revue, publiée en trois langues (anglais, espagnol, français), a des correspondants et des bureaux dans le monde entier 
(Belgique, Canada, Chili, Colombie, Espagne, France, GrandeBretagne, Italie, Luxembourg, Paraguay, Pays-Bas, Suisse...) et des rubriques y reviennent régulièrement: "Le film à l'école», "L’enfant au cinéma», «L'effort des catholiques».

En dépouillant ce périodique, on est frappé par la présence en son sein de toute une partie de la critique française (Henri Agel, Jean-George Auriol, Amédée Ayfre, André Bazin, Jean-Pierre Chartier - responsable de la version française de la revue Pierre Leprohon, Charles Ford, Jean-Louis Tallenay, Lo Duca...), qui recoupe à la fois La Revue du cinéma et, plus tard, les Cahiers du cinéma (mentionnés comme comportant de nombreux chrétiens, revue "alliée", en quelque sorte) et RadioCinéma-Télévision (futur Télérama). Si l'on ajoute l'influente collection $" 7^{\mathrm{e}}$ art " des éditions du Cerf, on mesure la place qu'occupe la pensée catholique dans le discours «savant" sur le cinéma. Incidemment, il pourrait être intéressant d'envisager l'éclairage qu'apporte cette appartenance sur la pensée de Bazin, sur le réalisme ontologique, sur la liberté du spectateur... La problématique du saint suaire occupe tout un dossier dans le n 5 (1950), «Numéro spécial de l'Année sainte» réalisé par les soins de Lo Duca, qui comprend également deux clichés pleine page de l'"empreinte correspondant à un négatif photographique» et un article sur "La photographie du Christ» — l'expression avait été utilisée auparavant par Claudel —, relatif à un film de Baldi consacré à la relique ${ }^{1}$.

En 1949, le no 2 de la revue est intitulé «À la recherche d'une doctrine chrétienne du cinéma». Parmi les contributions apportées à ce numéro, citons celles de Michel de Saint-Pierre ( «La querelle des intellectuels à propos du cinéma»), André Bazin («Misère, servitude et grandeur de la critique de films»), Pierre Leprohon et Jean-Pierre Chartier ( $"$ Le critique, le formalisme et la morale»), les déclarations des "professionnels du film français» (Fourré-Cormeray du CNC, Marcel L'Herbier ["Le film libre»], Jean-George Auriol [ «... et circenses»], Maurice Cloche, Pierre Fresnay, Pierre Blanchar, Morienval [" Apport chrétien à l'histoire du cinéma français»]). Abel Gance évoque un projet de film en ces termes - on relèvera en particulier le "finale" de son exhortation: 
C'est notre mission, c'est la mission transfiguratrice de notre Art, en ce tragique carrefour de l'Histoire Mondiale de 1949, de brandir au milieu de la tempête présente l'immense linceul du Christ comme un oriflamme, comme un drapeau de ralliement, drapeau sacré sur lequel doit s'inscrire en signes de feu sa Passion et son holocauste.

Et pendant que sa Passion se déroulera sous nos yeux, il nous faudra tous, autant que nous sommes, monter en même temps que Lui notre Calvaire personnel si nous voulons apaiser nos maux dans le miroir de ses souffrances.

Ce film [La divine tragédie] n'a pas d'autre but que d'aider les malheureux à gravir, les yeux levés, les premières marches qui montent au ciel. [...] Ainsi pourra s'établir pour la première fois dans le monde, sous le signe de la Croix du Christ, la chaîne entre tous les Chrétiens, entre tous ceux qui auront compris, selon les propres paroles de Pie XI, que le cinéma est le moyen le plus efficace d'influencer les masses.

Mais le croisement avec la filmologie est explicité par André Bazin quand il définit les tâches de la critique:

La naissance et le développement rapide du Centre international de filmologie est un signe précurseur. Non certes que le critique de demain se devra d'être filmologue, mais il ne pourra pas ignorer ces recherches... ( $n^{\circ} 2,1949$, p. 20)

Dès le $\mathrm{n}^{\circ} 5$, une contribution d'Agostino Gemelli (président de l'Académie pontificale des Sciences et recteur de l'Université catholique du Sacré-Cœur à Milan) expose le sujet très "filmologique", "Cinéma et psychologie», et se réfere à la "nouvelle science" ainsi qu'aux travaux de Cohen-Séat, Michotte, Wallon, Zazzo, Merleau-Ponty, etc. Gemelli marque bien, en outre, combien il fut lui-même un précurseur en la matière avec un article paru en 1928 dans le Journal de psychologie normale et pathologique ("Intérêt des projections cinématographiques»). Son approche, qui inscrit le cinéma avant tout comme "art du temps», insiste sur deux aspects: d'une part, sur le fait que dans le spectacle cinématographique, le "spectateur n'est pas, comme au théâtre, absent à ce qui arrive ou à ce que l'on représente; il est présent et suit la succession des épisodes en ayant conscience d'être présents à eux». D'autre part, il affirme que le film «actuel» «nous laisse la liberté de l'interpréter, de le "comprendre"... Le cinéaste n'est pas 
là pour nous dire ce qui arrive [...] le cinéaste moderne a appris cet art psychologique qui consiste à faire voir la signification et la suite des événements à travers des riens. [...] La présence du spectateur n'est pas passive. " À cet égard, Gemelli revoit à la lumière de l'évolution du cinéma les convictions qui étaient les siennes en 1928, époque durant laquelle il considérait que la projection plongeait le spectateur dans un état voisin d'un état onirique: "non-résistance de notre moi, atténuation des facultés critiques, projection de nos intérêts personnels».

Dans le $\mathrm{n}^{\circ} 12$ de la Revue internationale du cinéma (1952), Leo Lunders s'interroge: "Comment évaluer l'influence du cinéma sur les enfants?» (p. 50). Il écrit :

J'ai posé la question à M. Cohen-Séat :

- Nous ne pouvons encore rien dire à ce sujet.

— Quand le pourrez-vous?

- Peut-être dans dix ans, peut-être plus tard encore.

Nous ne pouvons pas attendre dix ans.

L'article se réfere aux enquêtes, questionnaires et tests publiés dans la Revue de filmologie (études de Zazzo, Gratiot-Alphandéry, Fulchignoni, enquêtes du Payne Fund). Dans un numéro suivant ( $\left.n^{\circ} 18,1954\right)$ consacré au "Cinéma et l'enfance», il revient à la "science filmologique» et à Cohen-Séat pour circonscrire le problème de l'influence du film sur le spectateur (p. 10-18).

Dans le no 15 (1953), Georges Damas (professeur à l'Idhec) rend compte de L'univers filmique, qu'a dirigé Souriau (p. 52-53), et commente: "Une équipe de chercheurs qualifiés s'est penchée sur les problèmes du cinéma et, dans les cadres de l'Institut de filmologie..." Il salue l'effort pour mettre au point un lexique précis et commente les contributions de Souriau, Riniéri et Agel.

Dans le n 16 (1953, p. 59), l'abbé Amédée Ayfre écrit que «toutes les études dont le cinéma peut être l'objet convergent actuellement sur l'Institut de filmologie...» Il signale en outre la soutenance de thèse de $\mathrm{M}^{\text {lle }}$ Poncet sur l'esthétique du dessin animé devant un "jury de filmologues» et se félicite de retrouver dans les colonnes de la Revue internationale de filmologie (RIF) des spécialistes éminents, "et parmi eux un certain nombre de chrétiens» (Gemelli, de Waehlens, Fulchignoni, Agel...). 
Au niveau international, si la participation des Canadiens demeure minime (un entrefilet mentionne l'existence de filmologues canadiens), on aura des articles sur la filmologie en Allemagne et en particulier à Munich ( $\left.\mathrm{n}^{\circ} 21,1955\right)$, mais ce sont les Italiens qui sont tout de suite partie prenante de la démarche filmologique et font état de l'écho qu'elle trouve dans leur pays :

La filmologie c'est-à-dire la recherche scientifique sur le cinéma et ses applications pénètre de plus en plus dans l'enceinte des Académies. Sous la direction de notre ami le Prof. Fulchignoni, l'Université de Rome a organisé une série de cours de filmologie.

Mario Verdone ( $\left.\mathrm{n}^{\circ} 4,1949\right)$, s'interrogeant sur l'approche critique du cinéma, accorde à la filmologie une place de choix : replacée dans la filiation de "pionniers" comme Luciano de Feo avec La Rivista del cinema educatore et Cinema, dans les années 1930, et comme Eisenstein, Epstein ou Arnheim, elle «envahit aujourd'hui toutes les branches du savoir: psychologie, sociologie, philosophie, linguistique, esthétique, histoire, morale, disciplines techniques, littérature, critique spécialisée (c'està-dire littérature cinématographique) et devient [...] la vraie science et philosophie du film» (p. 22-23).

Dans le $\mathrm{n}^{\circ} 13$ (1952, p. 41), Verdone évoque la formation des professionnels et des éducateurs en Italie et indique que le Centro Sperimental «a organisé des cours de filmologie pour les professionnels».

\section{Le $2^{\mathrm{e}}$ Congrès et Pie XII}

À l'occasion du $2^{\text {e }}$ Congrès international de filmologie de 1955 , la revue consacre un numéro complet au sujet $\left(\mathrm{n}^{\circ} 21\right.$, 1955) avec, en ouverture, un article intitulé "La commission pontificale pour le cinéma, la radio, la télévision", des informations sur la filmologie en Allemagne et un article sur une réunion de filmologues catholiques. C'est cependant le bilan de dix pages de la revue que dresse Ayfre qui aborde avec le plus de profondeur les apports et les limites des travaux du Congrès. L'auteur - qui déplore la trop faible place accordée à l'esthétique dans les approches utilisées (ainsi voit-il la réflexion d'Edgar Morin sur «la 
structure de l'imaginaire et le réalisme des films», heureusement complétée par les considérations plus directement esthétiques du R.P. Lunders) - s'interroge en conclusion sur la possibilité de voir converger les diverses disciplines théoriques et pratiques, car:

la constitution d'une Filmologie digne de ce nom est à ce prix, sinon les savants s'enfermeront dans leur point de vue propre et continueront à faire de la physiologie, de l'esthétique ou de la sociologie, les praticiens des films de l'éducation cinématographique, et la filmologie ne sera qu'une case vide réunissant artificiellement des éléments hétéroclites.

En 1956, dès lors, devant l'" Exhortation de Pie XII au monde du cinéma", le rédacteur en chef de la revue, Pierre d'André (par ailleurs critique cinématographique à Ouest-France, à l'Essor et à la Croix du Midi) rappelle qu' "en février $1955 \mathrm{a} \mathrm{eu}$ lieu à Paris le $\mathrm{II}^{\mathrm{e}}$ Congrès international de filmologie» et s'indigne d'y avoir

constaté avec stupeur l'absence quasi générale des critiques cinématographiques dont certains ne nous ont pas caché leur indifférence pour des recherches «sans aucun rapport avec les impératifs de leur profession et sans aucune utilité pour eux». Si cette attitude postule la non-influence du film, nous explique le dédain poli de ces mêmes critiques pour la cotation morale, elle nous fait toucher du doigt la légèreté de certains journalistes, esthètes avant tout, qui ne réalisent pas qu'ils jouent avec une bombe atomique d'un nouveau genre et que cette bombe atomique, le cinéma, est fort capable de désintégrer la personnalité humaine! Puisse le discours de SS Pie XII leur faire prendre conscience de leur erreur!

C'est donc tout naturellement qu'il sollicite de Cohen-Séat «le point de vue du filmologue» sur les récentes interventions du pape:

M. Gilbert Cohen-Séat a consacré une extrême attention à l'analyse des deux Discours que sa Sainteté a prononcés les 21 juin et 28 octobre $1956^{2}$.

Le Directeur de l'Institut de filmologie de l'Université de Paris considère en effet que ces Discours soulèvent une masse d'idées et d'observations à la fois par leurs textes mêmes et, pour ainsi dire, par ce que les textes contiennent "entre les lignes». 
C'est en effet la première fois qu'une instance de cette importance traite, avec cette ampleur, des problèmes du cinéma. Jusqu'à ce jour, deux "souverains" seulement avaient abordé cette question: Lénine ${ }^{3}$ et Pie XI. Mais l'encyclique Vigilanti Cura avait surtout un caractère préventif résultant d'une première analyse étendue qui était, logiquement, celle des dangers $\mathrm{du}$ film. Cette position du Saint-Siège représentait une étape d'urgence nécessaire dans le pragmatisme ${ }^{4}$.

Notre interlocuteur observe que le Pape, avec ses deux discours de 1955, franchit une deuxième étape.

Au souci de prévention et de mise en garde succède l'affirmation des aspects positifs du cinéma. Voici cette "institution" présentée comme un tout positif, un avenir, une puissance qui apporte quelque chose. Dès lors se trouve posé, pour le SaintPère et pour ceux dont il est le chef, le "comment" positif du cinéma. Cette préoccupation, essentiellement constructive, amène le Souverain Pontife à intervenir sous une forme dont notre interlocuteur souligne tour à tour la vigueur et la compréhension. Le seul mot qui vienne à l'esprit, nous dit M. CohenSéat, est celui de médiation. Se livrant à une vaste synthèse, recherchant les points communs, le Pape tente de mettre d'accord la multitude des points de vue à propos du cinéma. Et il y parvient! Mais précisément ce qui distingue la médiation du simple compromis, ce n'est pas seulement l'élévation de pensée et la rectitude intellectuelle, c'est surtout une pénétration fondée sur les aspects positifs des thèses en présence; c'est un accord, une concession.

Enfin M. Cohen-Séat est convaincu qu’avant peu Pie XII franchira la troisième étape.

Après avoir dénoncé les dangers du cinéma, après en avoir souligné les aspects constructifs, après l'avoir présenté comme un «tout» immense, le pape, qui n'a pas omis d'évoquer longuement des recherches scientifiques dont le film est l'objet, en viendra infailliblement à conduire les perspectives d'une connaissance objective des effets du cinéma. Puisqu'il existe des lois qui régissent l'emprise du cinéma sur l'individu, il faut trouver ces lois et il faut les étudier. L'ère de l'amateurisme, en pareille matière, est définitivement révolue. S'il faut un "Codex", ce codex n'est pas et ne peut pas être ramené à la Censure, ou en tout cas ne peut pas n'être défini que par la Censure. Celle-ci n'est qu'une forme d'empirisme nécessaire, mais insuffisant. Seule la recherche scientifique peut permettre d'établir des critères et des normes.

On lit entre les lignes l'inquiétude du premier Pasteur de la Chrétienté à l'idée que tout un pan de la "civilisation écrite» se 
trouve menacé de désuétude alors que c'est jusqu'ici dans ce cadre de l'écrit et de l'enseigné que se sont trouvées contenues à la fois les forces et les faiblesses de la tradition et de l'endoctrinement.

Peut-être était-il trop tôt pour bousculer la résistance tenace d'une certaine "intelligentsia" qui s'accroche à ce qu'elle a baptisé Septième Art. Le Pape s'est abstenu en effet, et ce n'est peut-être pas sans dessein, de parler de ces «élites» qui se sont toujours attribué un rôle majeur dans le développement des civilisations et qui, pour la première fois, sentent bien que, loin d'assurer la direction du mouvement, elles ne parviennent même plus à y participer tout à fait.

Le cinéma, ajoute $M$. Cohen-Séat, comporte dans son essence cette notion essentielle du "religare" qui fait que la conception du film idéal tel que le Pape en a brossé le tableau rejoint si bien la notion de "catholicité" dans son sens primitif et premier ${ }^{5}$.

Ces "recherches scientifiques" dont a parlé le pape ne sont autres que les recherches effectuées dans le domaine de la filmologie. Voici le texte même de Pie XII :

Mais pour pénétrer toute l'efficacité du film et pour apprécier à sa juste valeur la cinématographie, il faut porter son attention sur la part importante qu'y tiennent les lois de la psychologie, soit pour expliquer la manière dont le film agit sur les esprits, soit qu'on s'en serve consciemment pour faire plus d'impression sur les spectateurs. Les spécialistes de cette science observent avec soin le processus d'action et de réaction que suscite la vision du film, en appliquant la méthode d'enquête, l'analyse, les résultats de la psychologie expérimentale, et, [en] scrutant les couches secrètes du subconscient et de l'inconscient. Non seulement ils recherchent l'influence du film en tant que reçu passivement par le spectateur, mais ils analysent également sa puissance connexe d'activation psychique, selon ses lois immanentes, c'est-à-dire le pouvoir qu'il a de subjuguer un esprit par le charme de la représentation.

Si le pape ne nomme pas ici explicitement la filmologie, il le fait dans son deuxième discours $\left(2^{\mathrm{e}}\right.$ partie, 3$)$, "La réponse aux légitimes désirs ") : "Pour se faire une idée de la question, il n'est pas nécessaire de reprendre les considérations de filmologie et de psychologie dont Nous nous sommes déjà occupés...». 


\section{NOTES}

1. Avant Bazin et ses disciples, Girard-Cordonnier et Paul Claudel avaient vu dans le saint Suaire «la photographie du Christ» ( $c f$. Paul Claudel, «La photographie du Christ» [lettre à Girard-Cordonnier en réponse à l'envoi par celui-ci de son livre $L e$ Christ dans sa passion révélée par le saint-Suaire de Turin] dans Toi, qui es-tu?, Gallimard, 1936. Cité par Jérôme Thélot, Les inventions littéraires de la photographie, Paris, PUF, 2003, p. 89)

2. "Exhortations de Sa Sainteté le Pape Pie XII au monde du cinéma » $1^{\text {er }}$ discours (21 juin 1955) «Le pouvoir du cinéma»; $2^{\mathrm{e}}$ discours (28 octobre 1955) «Le film idéal efficace instrument d'élévation, d'éducation et d'amélioration ». Discours prononcés devant les représentants de l'industrie italienne et mondiale du film.

3. Rappelons que, pour ce qui est de Lénine, la question du cinéma tient en une courte phrase apocryphe "rapportée» après sa mort par Lounatcharski et qui devint un slogan à l'époque stalinienne

4. L'encyclique Vigilanti Cura n'a pas été le premier document pontifical concernant le cinéma. Un décret de la Sainte Congrégation Consistoriale publié en 1912 sous le pape Pie X la précède. Sept ans avant Vigilanti Cura, l'encyclique Divini ilius Magistri encourageait l'accès de la jeunesse à un cinéma qui «sans nuire à la vertu chrétienne pourrait au contraire l'enrichir" (Le cinéma dans l'enseignement de l'Église, Cité du Vatican, 1955, p. 6). Dans la lettre du futur pape Pie XII, alors cardinal secrétaire d'État, adressée au président de l'Office catholique international du cinéma en 1934, il était dit: "Les progrès scientifiques sont eux aussi des dons de Dieu dont il faut se servir pour Sa Gloire et pour l'extension de Son Règne» (Ibid., p. 51). Le 10 mars 1948, S.S. Pie XII, s'adressant aux prédicateurs de carême et aux curés de Rome, approuvait "les courageux efforts pour la production de films religieux ayant en même temps une réelle valeur artistique». Le 10 septembre 1948, le Saint-Père s'exprima au sujet du film d'enseignement dans son discours aux participants $\mathrm{du}$ Congrès national de l'Association italienne des enseignants catholiques. En 1951, le radio-message de Noël de Pie XII stigmatisait l'abandon de bon nombre à la presse, à la radio, au cinéma et à la télévision en guise de nourritures spirituelles. Notons que si Vigilanti Cura s'adressait aux évêques des États-Unis (à l'occasion d'une campagne de l'épiscopat catholique de ce pays pour entreprendre la "purification du cinéma" [Discours de S. E. M ${ }^{\text {gr }}$ le délégué apostolique du $1^{\text {er }}$ octobre 1933]) et du monde, les discours de 1955 s'adressent, eux, aux professionnels.

5. Revue internationale du cinéma, $\mathrm{n}^{\text {os }}$ 24-25, septième année, 1956, p. 75-77. 


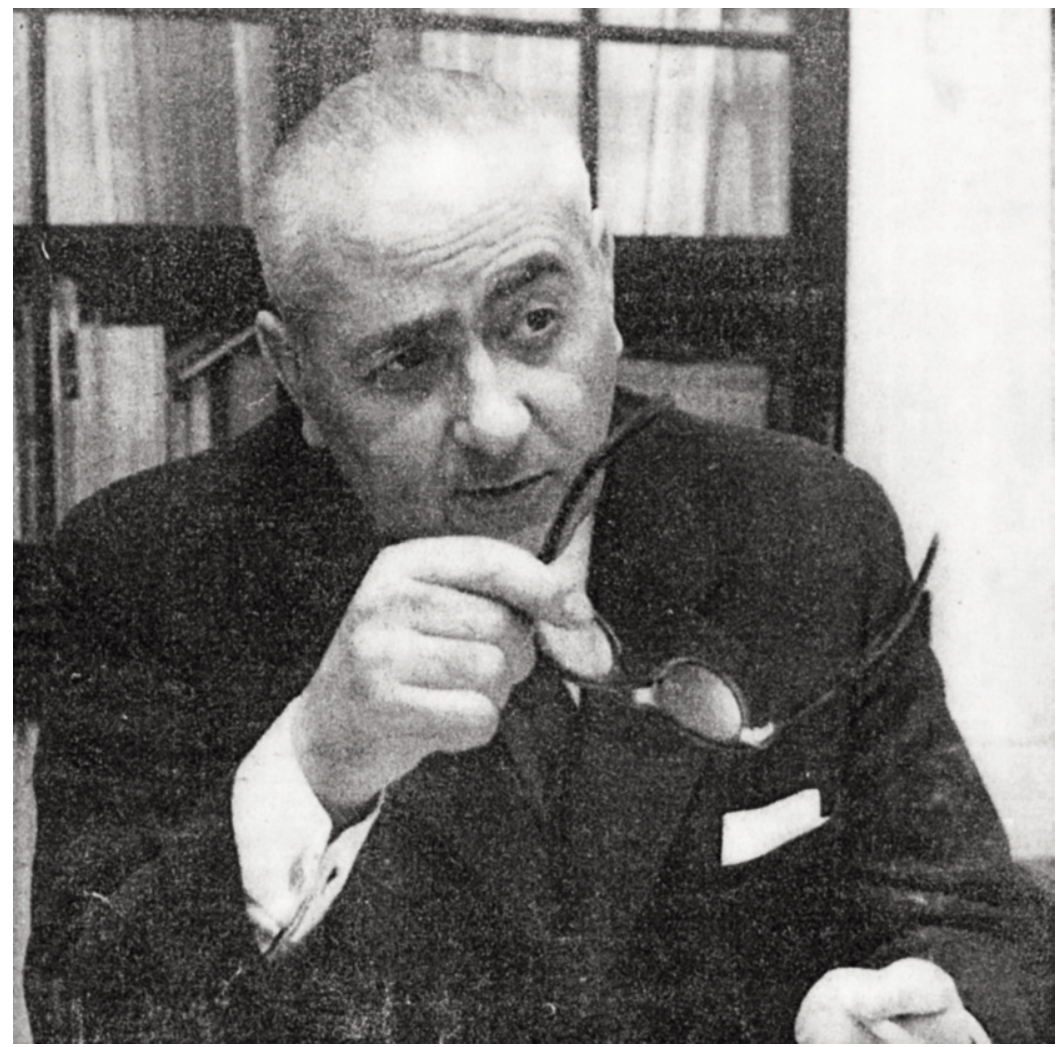

Portrait de Gilbert Cohen-Séat. Photo publiée dans L’Express du 5 avril 1962. (Photo: J.-R. Roustand) 